\title{
Quiste radicular infectado. Revisión de conceptos básicos y reporte de un caso clínico
}

\author{
Infected Radicular Cyst. \\ Review of Basic Concepts and A Clinical Case Report
}

Diego Rojas Jiménez DDS1; Daniela Zamora Arce2; Natalia Vargas Monge²; Diego Guillén Colombari DDS, MSc, Path ${ }^{3}$

1. Cirujano Oral y Maxilofacial, profesor adjunto de la Sección de Cirugía Oral y Maxilofacial de la Universidad de Costa Rica, Costa Rica.

2. Estudiante de sexto año, Facultad de Odontología, Universidad de Costa Rica, Costa Rica.

3. Médico patólogo, profesor adjunto al Departamento de Ciencias Quirúrgicas y Diagnósticas de la Universidad de Costa Rica, Costa Rica.

Autor para correspondencia: Dr. Diego Rojas Jiménez -diego.rojasjimenez@ucr.ac.cr

Recibido: 12-VII-2015

Aceptado: 28-X-2015

Publicado Online First: 6-XI-2015

DOI: http://dx.doi.org/10.15517/ijds.v0i0.21681

\section{RESUMEN}

Los quistes odontogénicos se dividen en quistes del desarrollo e inflamatorios. El quiste radicular o periapical, de origen inflamatorio, sigue siendo el quiste odontogénico más frecuentemente descrito y se forma a partir de restos de Malassez en el ligamento periodontal, como resultado de periodontitis apical posterior a la necrosis pulpar. El tratamiento de estas lesiones es variable, ya que en ocasiones es suficiente con un tratamiento endodóntico adecuado o en combinación con algunos procedimientos quirúrgicos (biopsia, marsupialización, descompresión). En los casos donde el tratamiento endodóntico inicial o retratamientos no resuelvan, se debe combinar con enucleaciones o apicectomías. A continuación se presenta un caso clínico de un masculino con lesión tumoral en mucosa oral a nivel de fondo de surco vestibular relacionado con la pieza 1.1. Se realiza estudio radiológico y se procede con la biopsia incisional, cuyo resultado reporta quiste periapical, por lo que se programa enucleación y curetaje de la lesión.

\section{PALABRAS CLAVE}

Quiste radicular, Quiste periapical, Granuloma periapical, Necrosis pulpar, Radiolucidez apical. 


\section{ABSTRACT}

Odontogenic cysts are divided into development and inflammatory cysts. Periapical or radicular cyst of inflammatory origin, remains the most frequently described odontogenic cyst and is formed from the epithelial rests of Malassez in the periodontal ligament as a result of apical periodontitis after pulpal necrosis. The treatment of these lesions is variable, sometimes the lesion is resolved with an endodontic treatment, or combination with certain surgical procedures (biopsy, marsupialization, decompression). However, in cases do not resolved with the initial endodontic treatment or retreatment, which should be combined with enucleation, apicoectomy. It is presented bellow a clinical case of a male patient with a tumor lesion on the vestibular sulcus in oral mucosa associated with dental organ 1.1. Radiological study was performed, it proceeds with incisional biopsy resulting reports periapical cyst, surgically enucleation and curettage of the lesion was performed.

\section{KEYWORDS}

Radicular cyst, Periapical cyst, Periapical granuloma, Pulp necrosis, Apical radiolucencies.

\section{INTRODUCCIÓN}

En 1974 Kramer et al. (1) definieron quiste como una cavidad patológica que contiene fluido, semifluido o gas y que no está formado por la acumulación de pus (2). Actualmente, se define como una cavidad recubierta por epitelio, el cual contiene en su luz material líquido, semisólido, sólido o combinación de las anteriores.

Los quistes odontogénicos se dividen en inflamatorios y del desarrollo. El quiste radicular pertenece a los quistes odontogénicos de origen inflamatorio junto con el quiste residual y el quiste paradental, según Shear y Speight (2).

A pesar de la dificultad por estandarizar los estudios, el quiste radicular sigue siendo, por mucho, el quiste odontogénico más frecuentemente descrito (2-3). Representa, aproximadamente, el $56 \%$ de las lesiones quísticas que afectan los maxilares, seguido del quiste dentígero y el tumor odontogénico queratoquístico $(2,4)$.

Los quistes radiculares se forman a partir de residuos epiteliales (restos de Malassez) en el ligamento periodontal, como resultado de periodontitis apical posterior a la necrosis pulpar, aunque también se pueden desarrollar a partir de epitelio crevicular, epitelio sinusal o recubrimiento epitelial de un tracto fistuloso (5-6-7).

\section{CARACTERÍSTICAS CLÍNICAS Y RADIOGRÁFICAS}

Típicamente, son asintomáticos, a menos que sufran una exacerbación inflamatoria aguda, la movilidad dental es posible cuando el quiste alcanza un tamaño importante, puede haber obliteración del fondo de saco vestibular asociado a la pieza dental involucrada. Cuando cursan con un proceso infeccioso crónico es posible que presenten tractos fistulosos con descarga activa de exudado fibrino-purulento, cursando clínicamente con exacerbaciones, generando eritema y edema en la encía y mucosa adyacente.

De acuerdo con Rosenberg et al. (8) dentro de los criterios utilizados para evaluar y diagnosticar, radiográficamente, una radiolucidez periapical como quiste, se encuentran: bordes corticalizados uniformes y bien definidos, resorción ósea alrededor de los dientes adyacentes, de forma ovalada o globo, lumen con radiolucidez uniforme, perforación de la tabla cortical, desplazamiento de raíces dentales adyacentes, tamaño mayor a $20 \mathrm{~mm}$, múltiples dientes involucrados 
periapicalmente, asociación de la lesión del ápice del diente involucrado, que se encuentre en maxila y en área de incisivos y caninos principalmente.

Radiográficamente, es idéntico a un granuloma periapical, lesión considerada como el origen de la formación del quiste radicular (9). Pueden coexistir traslapes de imagen entre un granuloma periapical amplios con imágenes de cavidades quísticas pequeñas por lo que no se puede realizar un diagnóstico definitivo mediante radiografía (2).

A pesar de que los quistes periapicales presentan, por lo general, un mayor tamaño que los granulomas, tanto la forma como el diámetro no deben ser utilizados como criterios diagnósticos definitivos (7).

Según Vier y Figueiredo (10-11), la reabsorción radicular suele ocurrir aunque no se aprecie en la radiografía, ya que la porción afectada es cemento en la mayoría de los casos. Sin embargo, los estudios no encontraron ninguna diferencia en la incidencia de la reabsorción en casos de granulomas y quistes radiculares.

\section{CARACTERÍSTICAS HISTOPATOLÓGICAS}

El quiste periapical está recubierto por un epitelio escamoso estratificado, el cual puede presentar exocitosis, espongiosis o hiperplasia, también es posible encontrar escasas células mucosas 0 áreas de epitelio respiratorio. En ocasiones, el epitelio tiene calcificaciones lineales 0 circulares conocidas como cuerpos de Rushton (7).

Este recubrimiento puede ser discontinuo y tener una gama de espesor de 1 a 50 capas de células. En su mayoría, el grosor es de 20 capas de células. La cobertura epitelial prolifera asociado a un proceso inflamatorio intenso o es inactivo y bastante regular con un cierto grado de diferenciación (12).
Según Shear y Speight (2), las paredes de la lesión varían de extremadamente delgadas a un grosor de $5 \mathrm{~mm}$ y la superficie interna es suave 0 corrugada. El fluido interno se puede observar de color café, como resultado de la presencia de sangre y cristales de colesterol, los cuales se observan de un color dorado brillante (13).

El infiltrado celular inflamatorio en los bordes epitelialesproliferativosconsistepredominantemente en leucocitos poliformonucleares, mientras que el infiltrado inflamatorio de la cápsula fibrosa adyacente consiste en infiltrado inflamatorio celular crónico $(2,14-15)$.

El lumen puede estar lleno de fluido, detritos celulares, calcificaciones distróficas, células gigantes multinucleadas, eritrocitos, hemosiderina, entre otros. La pared quística está compuesta de tejido conectivo fibroso denso, con infiltrado inflamatorio que contiene una mezcla variable de linfocitos, neutrófilos, células plasmáticas, histiocitos y eosinófilos (7).

Aproximadamente, el $10 \%$ de los quistes radiculares contienen cuerpos hialinos en los bordes epiteliales, los cuales se caracterizan por su apariencia rosa vítrea (hialinizada). Se cree que el origen de los cuerpos hialinos se relaciona con una hemorragia previa dentro de la pared del quiste inflamado (2).

Otra hipótesis, de acuerdo con Jacob (16) y Shamsudeen et al. (12), consiste en que el debris celular y los cristales de colesterol presentes en el quiste estimulan a las células a secretar sustancias amorfas, que luego pueden calcificarse, originando cuerpos hialinos de Rushton.

Según Meghji et al. (17), existen altos niveles de endotoxinas en quistes radiculares comparados con otros tipos de quistes como el folicular y el queratoquiste. Las bacterias Actinobacillus 
actinomycetemcomitans, Porphyromonas gingivalis y Escherichia coli podrían, directamente, estimular la proliferación epitelial de una manera dosis - dependiente. Los estudios proponen que las endotoxinas derivadas de la pulpa necrótica son el factor clave de iniciación de la patogénesis del quiste radicular.

\section{TRATAMIENTO}

El tratamiento de estas lesiones es variable, ya que en ocasiones es suficiente con un tratamiento endodóntico adecuado o combinación con algunos procedimientos quirúrgicos (biopsia, marsupialización, descompresión). Sin embargo, en algunos casos, la lesión no se resuelve con el tratamiento endodóntico inicial o con retratamientos, por lo que se debe combinar con enucleaciones y/o apicectomías (18).

En situaciones donde se presenta destrucción dental severa con lesión periodontal avanzada, la extracción del órgano dentario en cuestión puede llegar a ser obligatoria (como en este caso clínico).

Existe evidencia clínica indirecta de que los quistes radiculares retroceden después de un tratamiento endodóntico no quirúrgico, debido a que un alto porcentaje de dientes con periodontitis apical sanan luego de realizar dicha terapia. En otros casos, es menos probable la regresión de dicha lesión después del procedimiento endodóntico no quirúrgico, debido a que ya no depende de si existe o no infección en el conducto radicular. Según los resultados del estudio histopatológico de conductos radiculares tratados, se evidencia que se debe realizar el tratamiento quirúrgico en dichos casos (19).

Un punto importante que se debe considerar es que, en muchas ocasiones, estas lesiones periapicales no son sometidas a estudio histopatológico y se han visto casos en los cuales, a pesar de que representan en su mayoría lesiones benignas, mimetizan lesiones de comportamiento agresivo como tumores odontogénicos queratoquísticos y quistes odontogénicos ortoqueratinizado (20).

El estudio microscópico es imprescindible para un correcto diagnóstico en este tipo de lesiones, se debe utilizar para cualquier tejido suave removido durante procesos quirúrgicos de esta índole (20). Afortunadamente, todas aquellas lesiones inflamatorias que se remueven de forma completa (y se resuelve su causa) como los quistes periapicales, no recurren.

\section{DESCRIPCIÓN DEL CASO CLÍNICO}

Un paciente masculino de 32 años de edad acude a la Facultad de Odontología de la Universidad de Costa Rica, presentando dolor en región de incisivos maxilares de forma espontánea. Clínicamente se observa un aumento de volumen en región maxilar anterior con obliteración parcial del surco vestibular, de aproximadamente $2 \mathrm{~cm}$, con ligera crepitación; incisivo dental superior derecho con caries extensa, movilidad grado III, doloroso a la palpación y percusión, presencia de fístula en vestíbulo (Figura 1).

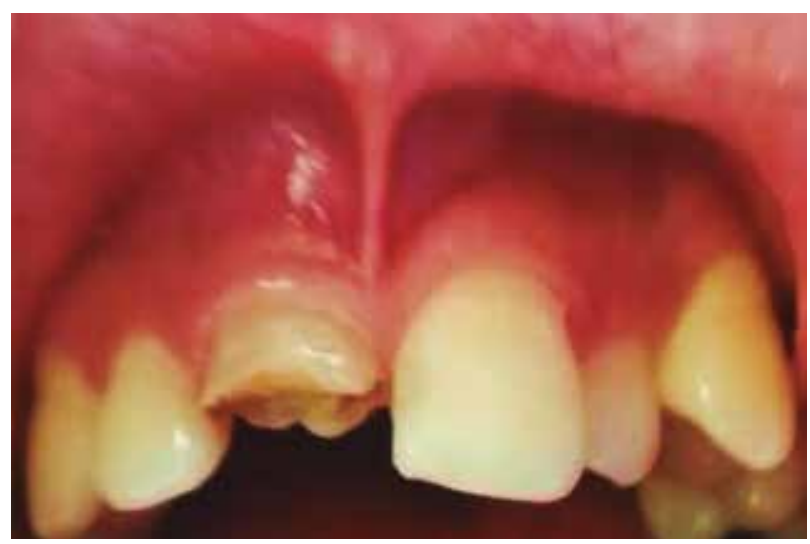

Figura 1. Se observa OD 1.1 con pérdida de sustancia dental, caries extensa, aumento de volumen en región maxilar anterior, con obliteración parcial del surco vestibular.

Se toma radiografía periapical, observando una imagen radiolúcida asociada a raíz de órgano 
dental (OD) 1.1, de $2.5 \mathrm{~cm}$ aproximadamente, unilocular, no corticalizado, bordes poco definidos, que se extiende hacia el piso nasal y hacia OD 1.2 a 2.1 (Figura 2).
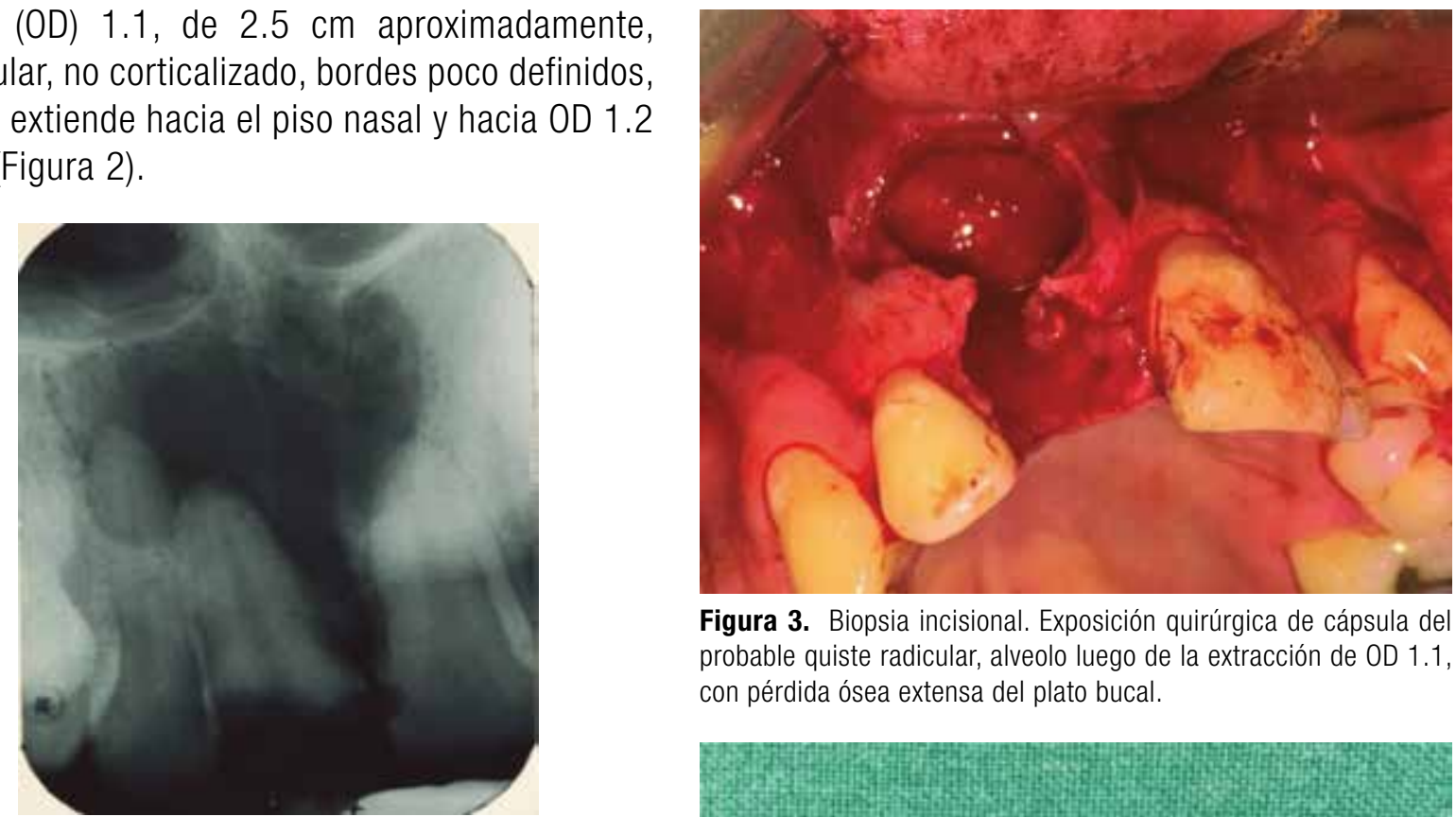

Figura 3. Biopsia incisional. Exposición quirúrgica de cápsula del probable quiste radicular, alveolo luego de la extracción de OD 1.1, con pérdida ósea extensa del plato bucal.

Figura 2. Radiografía periapical en la cual se observa imagen radiolúcida asociada a OD 1.1.

Se programa para biopsia, enucleación y curetaje de la lesión (figura 3), sin embargo, debido a que en el interior de la cavidad se observan pequeños depósitos similares a queratina, sospechando de un tumor odontogénico queratoquístico, se envía el espécimen (solo una porción de la cápsula) al laboratorio de patología que reporta quiste periapical. Se reprograma para enucleación y curetaje de la lesión de forma completa (figura 4), más extracción de OD 1.1, sin posibilidad de rehabilitación y sin soporte óseo en ninguna de las paredes del proceso alveolar (figura 5).

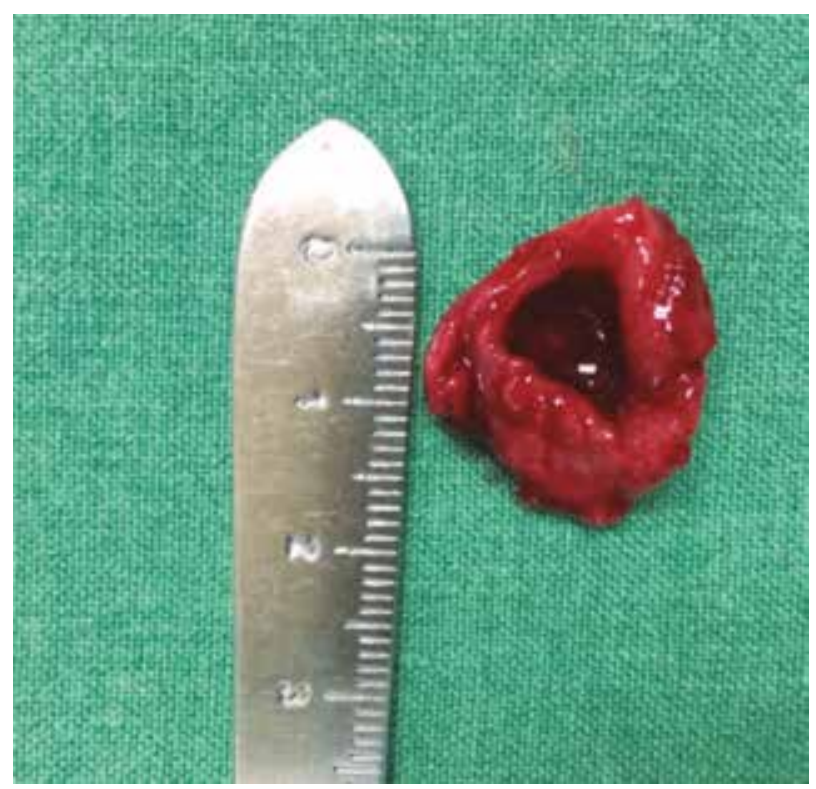

Figura 4. Cápsula del quiste radicular engrosada. 


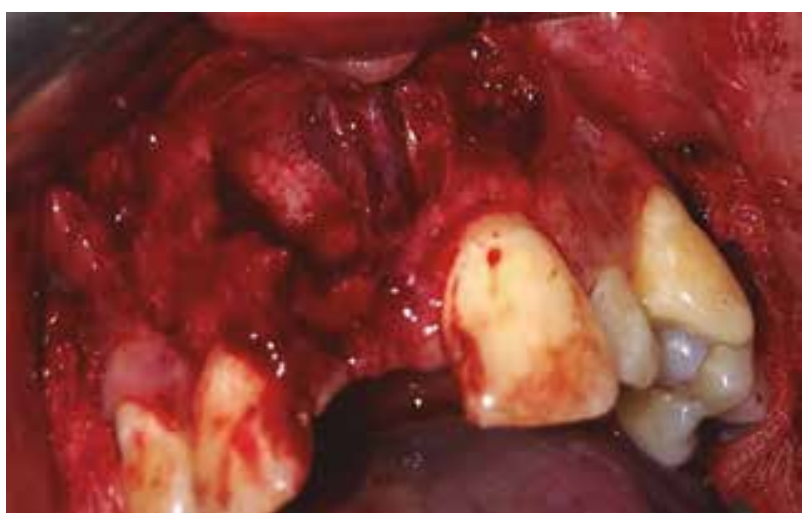

Figura 5. Defecto óseo secundario a enucleación y curetaje de quiste, se observa al fondo del lecho quirúrgico el paquete vásculonervioso nasopalatino íntegro.

Bajo anestesia local, se diseñó un colgajo trapezoidal de espesor completo, se suturó con puntos simples con catgut crómico 3-0, cursando el procedimiento sin complicaciones transoperatorias 0 posteriores. La terapia farmacológica consistió en un antimicrobiano betalactámico como lo es amoxicilina de 500 miligramos, en esquema de tres veces al día por 7 días, así como terapia analgésica y antiinflamatoria con ibuprofeno de 600 miligramos cada 8 horas durante 5 días y paracetamol de 500 miligramos cada 6 horas por un periodo de 5 días. Se colocó una prótesis transitoria para cubrir las necesidades estéticas.

El reporte histopatológico final es de quiste radicular, descartando el tumor odontogénico queratoquístico, pues este amerita un manejo quirúrgico diferente al del quiste radicular (figura 6). Se presentó una adecuada evolución clínica, sin eventualidades, ni complicaciones. Debido al defecto óseo originado por la lesión, se plantea la posibilidad de regeneración ósea guiada, en un futuro cercano, para una posterior rehabilitación mediante implante dental.

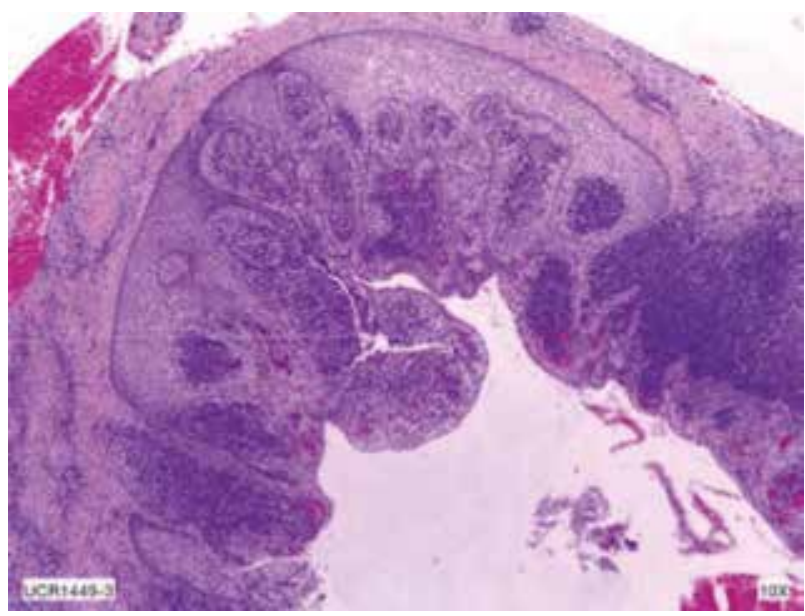

Figura 6. Se observa parte de la pared y el epitelio del quiste, con exudado inflamatorio agudo y crónico, el cual se localiza tanto en la pared como en el epitelio. 10X (Cortesía del Dr. Diego Guillén Colombari).

\section{DISCUSIÓN}

Los quistes periapicales o radiculares, estructuralmente, están formados por una pared de tejido conectivo, recubierta por epitelio con un lumen que contiene fluido o detritos celulares. Teóricamente, cada vez que el epitelio se descama en la luz quística, el contenido proteico se incrementa, generándose un aumento de tamaño, sin embargo, la mayoría de quistes periapicales crecen de forma lenta y asintomática a menos, que se infecten, donde la sintomatología dolorosa e inflamatoria se hace mayor de forma importante (2).

Dentro de los factores que pueden desencadenar la expansión quística se encuentra la proliferación epitelial, acumulación de contenidos celulares, crecimiento hidrostático, factores de resorción ósea y aumento de la actividad enzimática, los cuales se conjugan para incrementar el tamaño del quiste en forma progresiva (21-22). 
Respecto de la proliferación epitelial, el crecimiento periférico se atribuye a la división celular activa del revestimiento epitelial (21). Sin embargo, Toller en Mosqueda y Deister, aseguró que la división celular periférica a nivel de quistes radiculares no es la mejor justificación de su crecimiento por dos razones, en un tercio de los quistes radiculares existen áreas desprovistas de revestimiento epitelial y estos tienen un índice de actividad mitótica bajo, hasta siete veces menor respecto de los queratoquistes (21).

Según Kramer et al. (1), la producción y depósito de escamas de queratina en el lumen, como acumulación de contenido celular, representan áreas de expansión localizada con división celular aumentada, como en el caso de los queratoquistes, y precisamente este fue el caso donde durante la exploración quirúrgica se notó una probable presencia de queratina en la luz del quiste, por lo que se decidió realizar una biopsia incisional, para corroborar el diagnóstico definitivo.

El crecimiento hidrostático se basa en la distensión de la pared quística por el líquido acumulado. Esto se lleva a cabo por tres mecanismos: secreción, transudación - exudación y por último, diálisis. La secreción se evidencia por la presencia de células mucosecretoras en el revestimiento de los quistes (21).

Respecto de la transudación y exudación, Main (23) consideró que la exudación de un fluido rico en proteínas a través de los capilares se debe al efecto de la inflamación aguda. En 1979, Cohen (24) demostró, por medio de microfotografías, la presencia de canales intercelulares que atraviesan el espesor del epitelio quístico, permitiendo el paso del exudado inflamatorio de la cápsula al interior de la cavidad en quistes radiculares. Según Mosqueda y Deister (21), la presencia de fibrina y colesterol sugieren que la hemorragia favorece también la formación de fluido quístico.

En el proceso de diálisis, la osmolaridad promedio del líquido de quistes radiculares es más alta que la del suero, debido al acúmulo de proteínas de bajo peso molecular, la constante descamación epitelial y el acceso linfático inadecuado al lumen del quiste (21).

La resorción ósea es un requisito primordial para la habilidad de expansión de un quiste. Para ello, la vitamina $\mathrm{D}$, la hormona paratiroidea y las prostaglandinas son sustancias capaces de activar a los osteoclastos (21).

En el factor de actividad enzimática intracapsular se menciona la presencia de la colagenasa, sin embargo, se desconoce su papel en la expansión quística. Magnusson aseguró que también figura la leucina aminopeptidasa, implicada en la invasión de neoplasias malignas (21).

En este caso, la reabsorción ósea fue muy amplia a pesar de la poca sintomatología que presentó el paciente, así como a la naturaleza propia de la lesión la cual no alcanza, en forma general, esas dimensiones. Esto llevó a tomar la decisión de extraer el órgano dentario, debido a que no se contaba con soporte óseo alveolar, generando notable movilidad dental, lo que condicionó la permanencia del mismo.

El defecto óseo producido por el quiste se reconstruirá en una segunda fase quirúrgica, mediante regeneración ósea guiada y, posteriormente, la rehabilitación por medio de implantes dentales. El paciente evolucionó a la mejoría debido a la eliminación de los factores causales y en un lapso de 15 días ya no presentaba sintomatología alguna. 


\section{CONCLUSIONES}

El estudio histopatológico es una herramienta invaluable para el adecuado diagnóstico definitivo y manejo de lesiones periapicales, ya que existen otras patologías que pueden presentarse de forma similar.

Los métodos radiológicos no son determinantes en el diagnóstico de lesiones a nivel apical, ya que factores como la forma y el tamaño no son signos patognomónicos.

En casos de quistes radiculares verdaderos, la terapia endodóntica no es una opción por considerar de forma aislada, se debe combinar con enucleación y curetaje de la lesión.

Los quistes radiculares suelen ser asintomáticos, por lo que su detección temprana se va a dar con menos frecuencia, lo que puede permitir el crecimiento de la lesión durante un gran periodo

\section{REFERENCIAS}

1. Kramer IR., Pindborg J., Shear M. The WHO Histological Typing of Odontogenic Tumours. Cancer 70. 1992; 2988-2994.

2. Shear M., Speight P. M. Cysts of the oral and maxillofacial regions. Oxford, Inglaterra: Blackwell Munksgaard; 2007.

3. Koivisto T., Bowles W. R., Rohrer M. Frequency and distribution of radiolucent jaw lesions: a retrospective analysis of 9723 cases, J Endod. 2012; 38(6): 729-732.

4. Manor E., Kachko L., Puterman M., Szabo G., Bodner L. Cystic Lesions of the Jaws - A Clinicopathological Study of 322 Cases and Review of the Literature. Int. J. Med. Sci. 2012; 9(1): 20-26.
5. Nair P. N. Pathogenesis of apical periodontitis and the causes of endodontic failures. Crit Rev Oral Biol Med. 2004; 15(6): 348-381.

6. Lin L., Huang G. \& Rosenberg. Proliferation of Epithelial Cell Rests, Formation of Apical Cysts, and Regression of Apical Cysts after Periapical Wound Healing. J Endod. 2007; 33(8): $908-916$.

7. Neville B., Damm D., Allen C., Bouquot J. Oral \& Maxillofacial Pathology. Pensylvania, USA: W.B. Saunders Company. 2009. 1-194.

8. Rosenberg P., Frisbie J., Lee J., Lee K., Frommer H., Kottal S., Phelan J., Lin L., Fisch G. Evaluation of Pathologists (Histopathology) and Radiologists (Cone Beam Computed Tomography) Differentiating Radicular Cysts from Granulomas. J Endod. 2010; 36(3).

9. Scalas D., Roana J., Boffano P., Mandras N., Gallesio C., Amasio M., Banche G., Allizond V., Cuffini A. Bacteriological findings in radicular cyst and keratocystic odontogenic tumour fluids from asymptomatic patients. Archives of Oral Biology. 2013; 58: 1578-1583.

10. Vier F.V., Figueiredo J. A. P. Internal apical resorption and its correlation with the type of apical lesion. Int Endod J. 2004; 37: 730-737.

11. Vier F.V., Figueiredo J. A. Prevalence of different periapical lesions associated with human teeth and their correlation with the presence and extension of apical external root resorption. Int Endod J. 2002; 35: 710-719.

12. Shamsudeen M., Selvakumar T., Maguesh U., Prasad S., Kumar N. Radicular cyst a case report with an overview on pathogenesis. Indian J Multidiscip Den. 2013; 3(4): 824-827.

13. Rajendran B., Sivapathasundaram B. Cysts and Tumors of Odontogenic Origin. En Rajendran A., Sivapathasundaram B., Shafer's Textbook of Oral pathology. 2013. Cap 4: 1-963.

14. Matsuo T., Ebisu S., Shimabukuro Y., Ohtake T., Okada H. Quantitative analysis of 
immunocompetent cells in human periapical lesions: correlations with clinical findings of the involved teeth. J Endod. 1992; 18(10): 497-500.

15. Fraga C., Alves R. L., Sousa A. A. et al. Th1 and Th2-like protein balance in yhuman inflammatory radicular cysts and periapical granulomas. J Endod, 2013; 39(4): 453-455.

16. Jacob, S. Rushton bodies or hyaline bodies in radicular cysts: A morphologic curiosity. Indian J Multidiscip Den. 2010; 53: 846-847.

17. Meghji S., Qureshi W., Henderson B., Harris $M$. The role of endotoxin and cytokines in the pathogenesis of odontogenic cysts. Archives of Oral Biology. 1996; 41(6): 523-531.

18. Tek M., Metin M., Sener I., Bereket C., Tokac M., Kazancioglu H., Ezirganli S. The predominant bacteria isolated from radicular cysts. Head \& Face Medicine. 2013; 9(25). 1-6

19. Lin L., Ricucci D., Lin J. \& Rosenberg P. Nonsurgical Root Canal Therapy of Large Cyst-like Inflammatory Periapical Lesions and Inflammatory Apical Cysts. J Endod. 2009; 35(5).

20. Servato JP., Vitorino Cardoso S., Caetano Parreira da Silva M., Scalon Cordeiro M., Rogerio de Faria P., Mota Loyola A. Orthokeratinized Odontogenic Cysts Presenting as a Periapical Lesion: Report of a Case and Literature Review. J Endod. 2014; 40(3): 455-458.

21. Mosqueda T. A., Deister M. A. Los factores de expansión en quistes odontogénicos. Práctica Odontológica. 1985; 6(7): 13-20.

22. Leyva R., Tapia L., Quezada D., Ortíz. Factores involucrados en el desarrollo y expansión del quiste periapical. Rev. Odont. Mex. 2006; 10(1): 36-41.

23. Main D. M. Epithelial jaw cysts: a clinicopathological reappraisal. Brit J Oral Surg. 1970; 8(2): 114-125.

24. Cohen M. Pathways of inflammatory celular exudate through radicular cyst epithelium: A light and scanning electron microscope study. J. Oral Pathol. Med. 1979; 8(6): 369-378. 